International Journal of Pure and Applied Mathematics

Volume 109 No. 2 2016, 245-256

ISSN: 1311-8080 (printed version); ISSN: 1314-3395 (on-line version)

url: http://www.ijpam.eu

doi: 10.12732/ijpam.v109i2.7

\title{
STUDY ON i-v FUZZY TRANSLATION AND MULTIPLICATION OF i-v FUZZY $\beta$-SUBALGEBRA
}

\author{
P. Hemavathi ${ }^{1}$, P. Muralikrishna ${ }^{2}$, K. Palanivel $^{3}$ \\ ${ }^{1}$ Department of Mathematics \\ Sri Venkateswara College of Engineering \& Technology \\ Thirupachur, 631203, INDIA \\ ${ }^{2} \mathrm{PG}$ and Research Department of Mathematics \\ Muthurangam Government Arts College (Autonomus) \\ Vellore, 632002, INDIA \\ ${ }^{3}$ Department of Mathematics \\ School of Advanced Sciences \\ VIT University, Vellore, 632014, INDIA
}

\begin{abstract}
In this paper, we discuss the notion of an Interval valued fuzzy translation of $\mathrm{i}-\mathrm{v}$ fuzzy $\beta$-subalgebra and investigate some of their basic properties.
\end{abstract}

AMS Subject Classification: 08A72, 03E72

Key Words: $\beta$-algebra, $\beta$-subalgebra, fuzzy $\beta$-subalgebra, i-v fuzzy $\beta$-subalgebra

\section{Introduction}

After the notion of fuzzy sets, Zadeh in $[9,10]$ made an extension of a fuzzy set by an interval valued fuzzy set (ie. a fuzzy set with an interval valued membership function). This interval valued fuzzy set is referred as an i-v fuzzy set and applied in various algebraic structures.

Iseki et al. [6] introduced two classes of abstract algebras: BCK-algebras and BCI-algebras. During 2002, Neggers et al. [7] discussed $\beta$-algebras. In 2013 Chandramouleeswaran et al. [3] dealt Fuzzy Translation and Fuzzy Mul-

Received: June 4, 2016

Published: September 8, 2016

${ }^{\S}$ Correspondence author (c) 2016 Academic Publications, Ltd.

url: www.acadpubl.eu 
tiplication in BF/BG-algebras. In 2014 [1] Aub Ayub Ansari et al. applied the Fuzzy Translation on Fuzzy $\beta$-ideals of $\beta$-algebra. Motivated by these in $[4,5]$, we introduced an interval valued fuzzy $\beta$ - sub-algebras of $\beta$-algebra and product on i-v fuzzy $\beta$-subalgebra. In [2], Barbhuiya focused the Fuzzy Translations and fuzzy multiplications of interval valued fuzzy BG-algebra. Recently Sujatha et al. [8] introduced the notion of intuitionistic fuzzy $\alpha$-translation on $\beta$-algebras. With all these ideas, in this paper, we discuss the notion on $\mathrm{i}-\mathrm{v}$ fuzzy translation of i-v fuzzy $\beta$-subalgebras.

\section{Preliminares}

In this section we recall some basic definitions needed for our work.

Definition 2.1. [7] A $\beta$-algebra is a non-empty set $X$ with a constant 0 and two binary operations + and - satisfying the following axioms:

1. $x-0=x$

2. $(0-x)+x=0$

3. $(x-y)-z=x-(z-y) \forall x, y, z \in X$.

Example 2.2. Let $X=\{0, a, b, c\}$ be a set with constant 0 and binary operation + and -are defined on $X$ by the following Cayley's table

\begin{tabular}{|c|c|c|c|c|}
\hline+ & 0 & $a$ & $b$ & $c$ \\
\hline 0 & 0 & $a$ & $b$ & $c$ \\
\hline$a$ & $a$ & $b$ & $c$ & 0 \\
\hline$b$ & $b$ & $c$ & 0 & $a$ \\
\hline$c$ & $c$ & 0 & $a$ & $b$ \\
\hline
\end{tabular}

\begin{tabular}{|c|c|c|c|c|}
\hline- & 0 & $a$ & $b$ & $c$ \\
\hline 0 & 0 & $c$ & $b$ & $a$ \\
\hline$a$ & $a$ & 0 & $c$ & $b$ \\
\hline$b$ & $b$ & $a$ & 0 & $c$ \\
\hline$c$ & $c$ & $b$ & $a$ & 0 \\
\hline
\end{tabular}

Then $(X,+,-, 0)$ is a $\beta$-algebra.

Definition 2.3. A non empty subset $A$ of a $\beta$-algebra $(X,+,-, 0)$ is called a $\beta$-subalgebra of $X$, if $\quad \forall x, y \in X$

1. $x+y \in A$

2. $x-y \in A$

Example 2.4. In the above example of the $\beta$-algebra $X$, the subset $\{0, b\},\{0, a\},\{0, c\}$ are $\beta$-subalgebra of $X$. But the subset $A=\{0, a, b\}$ is not a $\beta$-subalgebra of $X$, since $(a+b=c \notin A)$ 
Definition 2.5. [1] Let $\mu$ be a fuzzy set in a $\beta$-algebra $X$. Then $\mu$ is called a fuzzy $\beta$-subalgebra of $X$, if $\forall x, y \in X$

1. $\mu(x+y) \geq \min \{\mu(x), \mu(y)\}$

2. $\mu(x-y) \geq \min \{\mu(x), \mu(y)\}$

Definition 2.6. [10] An interval valued fuzzy set (briefly i-v fuzzy set) $A$ defined on $X$ is given by

$$
A=\left\{\left(x,\left[\mu_{A}^{L}(x), \mu_{A}^{U}(x)\right]\right)\right\} \quad \forall x \in X
$$

(briefly denoted by $A=\left[\mu_{A}^{L}, \mu_{A}^{U}\right]$ ), where $\mu_{A}^{L}$ and $\mu_{A}^{U}$ are two fuzzy sets in $X$ such that $\mu_{A}^{L}(x) \leq \mu_{A}^{U}(x) \quad \forall x \in X$.

Let $\bar{\mu}_{A}(x)=\left[\mu_{A}^{L}(x), \mu_{A}^{U}(x)\right] \forall x \in X$ and let $D[0,1]$ denotes the family of all closed subintervals of $[0,1]$. If $\mu_{A}^{L}(x)=\mu_{A}^{U}(x)=c$, say, where $0 \leq c \leq 1$, then we have $\bar{\mu}_{A}(x)=[c, c]$ which we also assume, for the sake of convenience, to belong to $D[0,1]$. Thus $\bar{\mu}_{A}(x) \in D[0,1] \forall x \in X$, and therefore the $\mathrm{i}$-v fuzzy set $A$ is given by

$$
A=\left\{\left(x, \bar{\mu}_{A}(x)\right)\right\} \quad \forall x \in X,
$$

where $\bar{\mu}_{A}: X \rightarrow D[0,1]$.

Now let us define what is known as refined mimimum(briefly rmim) of two elements in $D[0,1]$. We also define the symbols $" \geq ", " \leq "$, and $"=$ " in case of two elements in $D[0,1]$.

Consider two elements $D_{1}:=\left[a_{1}, b_{1}\right]$ and $D_{2}:=\left[a_{2}, b_{2}\right] \in D[0,1]$.

Then we have:

$$
\operatorname{rmin}\left(D_{1}, D_{2}\right)=\left[\min \left\{a_{1}, a_{2}\right\}, \min \left\{b_{1}, b_{2}\right\}\right] ;
$$

$D_{1} \geq D_{2}$ if and only if $a_{1} \geq a_{2}, b_{1} \geq b_{2}$.

Similarly we may have $D_{1} \leq D_{2}$ and $D_{1}=D_{2}$.

Remark 2.7. Let $D_{1}:=\left[a_{1}, b_{1}\right]$ and $D_{2}:=\left[a_{2}, b_{2}\right] \in D[0,1]$.Then

1. $D_{1} \leq D_{2} \Leftrightarrow a_{1} \leq a_{2} \quad \& \quad b_{1} \leq b_{2}$

2. $D_{1}=D_{2} \Leftrightarrow a_{1}=a_{2} \quad \& \quad b_{1}=b_{2}$

3. $D_{1}+D_{2}=\left[a_{1}+a_{2}, b_{1}+b_{2}\right]$ whenever $a_{1}+a_{2} \leq 1$ and $b_{1}+b_{2} \leq 1$

4. $D_{1}-D_{2}=\left[a_{1}-a_{2}, b_{1}-b_{2}\right]$ whenever $a_{1}-a_{2} \leq 1$ and $b_{1}-b_{2} \leq 1$

Definition 2.8. [4] Let $\bar{\mu}_{A}$ be an i-v fuzzy subset in $X$. Then $\bar{\mu}_{A}$ is said to be interval valued fuzzy(i-v-fuzzy) $\beta$-subalgebra of $X$, if $\quad \forall x, y \in X$ 
1. $\bar{\mu}_{A}(x+y) \geq \operatorname{rmin}\left\{\bar{\mu}_{A}(x), \bar{\mu}_{A}(y)\right\}$

2. $\bar{\mu}_{A}(x-y) \geq \operatorname{rmin}\left\{\bar{\mu}_{A}(x), \bar{\mu}_{A}(y)\right\}$

Example 2.9. Consider the $\beta$-algebra $X=\{0, a, b, c\}$ in example 2.2. Define an $\mathrm{i}-\mathrm{v}$ fuzzy subset $\bar{\mu}$ of $X$ defined by

$$
\bar{\mu}(x)= \begin{cases}{[0.3,0.7]:} & x=0 \\ {[0.1,0.5]:} & x=a, c \\ {[0.2,0.6]:} & x=b\end{cases}
$$

Then $\bar{\mu}$ is an $\mathrm{i}-\mathrm{v}$ fuzzy $\beta$-subalgebra of $\mathrm{X}$.

Definition 2.10. [1] Let $\mu$ be a fuzzy set of a $\beta$-algebra $X$ and $\alpha \in[0, T]$ where $T=1-\sup \{\mu(x) / x \in X\}$. Then the fuzzy set $\mu_{\alpha}^{T}: X \rightarrow D[0,1]$ is called a fuzzy $\alpha$-translation of $\mu$ if $\mu_{\alpha}^{T}(x)=\mu(x)+\alpha, \forall x \in X$.

\section{Interval Valued Fuzzy Translations of $\beta$-Subalgebra}

This section, deals with the notion of Interval valued fuzzy translation of $\beta$-subalgebra. In what follows, $X$ denotes a $\beta$-algebra and for any i-v fuzzy set $\bar{\mu}$ of $X$, we denote $\bar{T}=[1,1]-\operatorname{rsup}\{\bar{\mu}(x) / x \in X\}$ unless otherwise specified.we start with,

Definition 3.1. Let $\bar{\mu}$ be an i-v fuzzy set of $X$ and $\bar{\alpha} \in[\overline{0}, \bar{T}]$, where $\bar{\alpha}=\left[\alpha^{L}, \alpha^{U}\right]$ with $\alpha^{L} \in\left[0, T^{L}\right] \& \alpha^{U} \in\left[0, T^{U}\right]$ and $\overline{0}=[0,0]$. A mapping $\bar{\mu} \overline{\frac{T}{\alpha}}: X \rightarrow D[0,1]$ is said to be an i-v fuzzy $\bar{\alpha}$-translation of $\bar{\mu}$ if it satisfies $\bar{\mu} \overline{\bar{\alpha}}(x)=\bar{\mu}(x)+\bar{\alpha}, \forall x \in X$.

Example 3.2. Consider the $\beta$-algebra $X=\{0, a, b, c\}$ in example 2.2. Define an interval valued fuzzy subset $\bar{\mu}$ of $X$ by

$$
\bar{\mu}(x)= \begin{cases}{[0.3,0.7]:} & x=0 \\ {[0.1,0.5]:} & x=a, c \\ {[0.2,0.6]:} & x=b\end{cases}
$$

Then $\bar{\mu}$ is an i-v fuzzy $\beta$-subalgebra of $X$. Here $\bar{T}=[1,1]-r \sup \{\bar{\mu}(x) / x \in$ $X\}=[1,1]-[0.3,0.7]=[0.7,0.3]$. choose $\bar{\alpha}=[0.04,0.08] \in[\overline{0}, \bar{T}]$. Then the $\mathrm{i}-\mathrm{v}$ fuzzy set $\bar{\mu} \frac{\bar{T}}{\alpha}: X \rightarrow D[0,1]$ is given by $\bar{\mu} \frac{\bar{T}}{\alpha}(0)=[0.34,0.78], \quad \bar{\mu} \frac{\bar{T}}{\alpha}(a)=\bar{\mu}_{\bar{\alpha}}(c)=$ $[0.14,0.58]$ and $\bar{\mu} \overline{\frac{T}{\alpha}}(b)=[0.24,0.68]$ is a $\mathrm{i}-\mathrm{v}$ fuzzy $\bar{\alpha}-$ Translation of $\bar{\mu}$. 
Theorem 3.3. For any i-v fuzzy $\beta$-subalgebra $\bar{\mu}$ of $X$ and $\bar{\alpha} \in[\overline{0}, \bar{T}]$, the i-v fuzzy $\bar{\alpha}$-translation $\bar{\mu} \overline{\bar{\alpha}}(x)$ of $\bar{\mu}$ is an i-v fuzzy $\beta$-subalgebra of $X$.

Proof. Let $x, y \in X$ and $\bar{\alpha} \in[\overline{0}, \bar{T}]$, Then:

$$
\bar{\mu}(x+y) \geq \operatorname{rmin}\{\bar{\mu}(x), \bar{\mu}(y)\}
$$

and

$$
\bar{\mu}(x-y) \geq \operatorname{rmin}\{\bar{\mu}(x), \bar{\mu}(y)\}
$$

Now

$$
\begin{aligned}
\bar{\mu} \overline{\frac{T}{\alpha}}(x+y) & =\bar{\mu}(x+y)+\bar{\alpha} \\
& \geq \operatorname{rmin}\{\bar{\mu}(x), \bar{\mu}(y)\}+\bar{\alpha} \\
& =\operatorname{rmin}\{\bar{\mu}(x)+\bar{\alpha}, \bar{\mu}(y)+\bar{\alpha}\} \\
& =\operatorname{rmin}\left\{\bar{\mu}_{\bar{\alpha}}(x), \bar{\mu} \bar{\alpha}(y)\right\}
\end{aligned}
$$

Similarly, $\bar{\mu} \overline{\frac{T}{\alpha}}(x-y) \geq \operatorname{rmin}\left\{\bar{\mu} \frac{\bar{T}}{\alpha}(x), \bar{\mu} \frac{\bar{T}}{\alpha}(y)\right\}$

Hence $\bar{\mu} \bar{\alpha}$ of $\bar{\mu}$ is an i-v fuzzy $\beta$-subalgebra of $X$.

The following is the converse of the above theorem.

Theorem 3.4. For any $\mathrm{i}-\mathrm{v}$ fuzzy subset $\bar{\mu}$ of $X$ and $\bar{\alpha} \in[\overline{0}, \bar{T}]$. If the $\mathrm{i}-\mathrm{v}$ fuzzy $\bar{\alpha}$-translation $\bar{\mu} \frac{\bar{\alpha}}{\alpha}$ of $\bar{\mu}$ is also an i-v fuzzy $\beta$-subalgebra of $X$, then so is $\bar{\mu}$.

Proof. Let $x, y \in X$

Assume that $\bar{\mu} \bar{\alpha}(x)$ of $\bar{\mu}$ is a i-v fuzzy $\beta$-subalgebra of $X$ for some $\bar{\alpha} \in[\overline{0}, \bar{T}]$.

Then:

$$
\begin{aligned}
\bar{\mu}(x+y)+\bar{\alpha} & =\bar{\mu} \overline{\bar{T}}(x+y) \\
& \geq \operatorname{rmin}\left\{\bar{\mu} \frac{\bar{T}}{\bar{\alpha}}(x), \bar{\mu} \bar{\alpha}(y)\right\} \\
& =\operatorname{rmin}\{\bar{\mu}(x)+\bar{\alpha}, \bar{\mu}(y)+\bar{\alpha}\} \\
& =\operatorname{rmin}\{\bar{\mu}(x), \bar{\mu}(y)\}+\bar{\alpha}
\end{aligned}
$$

$\Rightarrow \bar{\mu}(x+y) \geq \operatorname{rmin}\{\bar{\mu}(x), \bar{\mu}(y)\}$.

Similarly, $\bar{\mu}(x-y) \geq \operatorname{rmin}\{\bar{\mu}(x), \bar{\mu}(y)\}$.

Hence $\bar{\mu}$ is an i-v fuzzy $\beta$-subalgebra of $X$. 
Remark 3.5. In general for any $\mathrm{i}-\mathrm{v}$ fuzzy set $\bar{\mu}$ of $X$, the $\mathrm{i}-\mathrm{v}$ fuzzy $\bar{\alpha}$-translation $\bar{\mu} \frac{\bar{T}}{\alpha}(\bar{\alpha} \in[\overline{0}, \bar{T}])$ of $\bar{\mu}$ need not be an i-v fuzzy $\beta$-subalgebra of $X$, as shown by the following example.

Let $X$ be the $\beta$-algebra given in Example 3.2. Consider the $\mathrm{i}-\mathrm{v}$ fuzzy set $\bar{\mu}$

$$
\bar{\mu}(x)= \begin{cases}{[0.4,0.6]:} & x=0 \\ {[0.3,0.5]:} & x=a \\ {[0.2,0.4]:} & x=b \\ {[0.1,0.3]:} & x=c\end{cases}
$$

Let $\bar{\alpha}=[0.02,0.03]$. Then the corresponding $\bar{\alpha}$-translation is

$$
\begin{gathered}
\bar{\mu} \bar{\alpha}(0)=[0.42,0.63], \quad \bar{\mu}_{\bar{\alpha}}^{T}(a)=[0.32,0.53] \\
\bar{\mu}_{\bar{\alpha}} \bar{T}(b)=[0.22,0.43] \text { and } \bar{\mu} \frac{\bar{T}}{\alpha}(c)=[0.12,0.33] .
\end{gathered}
$$

Now $\bar{\mu}(a+b)=\bar{\mu}(c)=[0.1,0.3] \ngtr[0.2,0.4]=\operatorname{rmin}\{\bar{\mu}(a), \bar{\mu}(b)\}$ and $\bar{\mu} \bar{\alpha}(a+b)=$ $\bar{\mu} \bar{\alpha}(c)=[0.12,0.33] \ngtr[0.22,0.43]=\operatorname{rmin}\left\{\bar{\mu} \overline{\frac{T}{\alpha}}(a), \bar{\mu} \overline{\bar{\alpha}}(b)\right\}$.

Hence $\bar{\mu}$ and $\bar{\mu} \bar{\alpha}$ are not i-v fuzzy $\beta$-subalgebra of $X$.

Corollary 3.6. Let $\bar{\mu}$ be an i-v fuzzy set of $X$. If $\bar{\alpha}=\overline{0}$ then the $\mathrm{i}-\mathrm{v}$ fuzzy $\bar{\alpha}$-translation $\bar{\mu} \bar{\alpha}$ of $\bar{\mu}$ is an i-v fuzzy $\beta$-subalgebra of $X$.

Theorem 3.7. Let $\bar{\mu}$ be given an i-v fuzzy $\beta$-subalgebra of $X$. Then for $\bar{\alpha}, \overline{\alpha^{\prime}} \in[\overline{0}, \bar{T}],\left(\bar{\mu} \frac{\bar{T}}{\bar{\alpha}} \cap \bar{\mu} \frac{\bar{T}}{\alpha^{\prime}}\right)$ and $\left(\bar{\mu} \frac{\bar{T}}{\bar{\alpha}} \cup \bar{\mu} \frac{\bar{T}}{\alpha^{\prime}}\right)$ are also an i-v fuzzy $\beta$-subalgebra of $X$.

Proof. Let $\bar{\mu} \frac{\bar{T}}{\alpha}$ and $\bar{\mu} \frac{\bar{T}}{\alpha^{\prime}}$ be two i-v fuzzy translation of an i-v fuzzy $\beta$-subalgebra $\bar{\mu}$ of $X$, where $\bar{\alpha}, \overline{\alpha^{\prime}} \in[\overline{0}, \bar{T}]$

Assume that $\bar{\alpha} \leq \overline{\alpha^{\prime}}$ by theorem $3.3 \bar{\mu} \frac{\bar{\alpha}}{\bar{\alpha}}$ and $\bar{\mu} \frac{\bar{T}}{\alpha^{\prime}}$ be two i-v fuzzy translation of $\beta$-subalgebra of $X$. Now

$$
\begin{aligned}
\left(\bar{\mu} \frac{\bar{T}}{\alpha} \cap \bar{\mu} \frac{\bar{T}}{\alpha^{\prime}}\right)(x) & =\operatorname{rmin}\left\{\bar{\mu} \frac{\bar{T}}{\bar{\alpha}}(x), \bar{\mu} \frac{\bar{T}}{\alpha^{\prime}}(x)\right\} \\
& =\operatorname{rmin}\left\{\bar{\mu}(x)+\bar{\alpha}, \bar{\mu}(x)+\overline{\alpha^{\prime}}\right\} \\
& =\bar{\mu}(x)+\bar{\alpha} \\
& =\bar{\mu}_{\bar{\alpha}}^{T}(x) .
\end{aligned}
$$

Also

$$
\left(\bar{\mu} \frac{\bar{T}}{\bar{\alpha}} \cup \bar{\mu} \frac{\bar{T}}{\alpha^{\prime}}\right)(x)=\operatorname{rmax}\left\{\bar{\mu} \frac{\bar{T}}{\alpha}(x), \bar{\mu} \frac{\bar{T}}{\alpha^{\prime}}(x)\right\}
$$




$$
\begin{aligned}
& =\operatorname{rmax}\left\{\bar{\mu}(x)+\bar{\alpha}, \bar{\mu}(x)+\overline{\alpha^{\prime}}\right\} \\
& =\bar{\mu}(x)+\overline{\alpha^{\prime}} \\
& =\bar{\mu} \frac{\bar{T}}{\alpha^{\prime}}(x)
\end{aligned}
$$

$\left(\bar{\mu} \overline{\bar{\alpha}} \cap \bar{\mu} \frac{\bar{T}}{\alpha^{\prime}}\right)$ and $\left(\bar{\mu} \frac{\bar{\alpha}}{\bar{\alpha}} \cup \bar{\mu}_{\bar{\alpha}^{\prime}}^{\bar{T}}\right)$ is an i-v fuzzy $\beta$-subalgebra of $X$.

Theorem 3.8. Let $\bar{\mu}_{1}$ and $\bar{\mu}_{2}$ be two i-v fuzzy $\beta$-subalgebras of $X$. Let $\bar{T}=\operatorname{rmin}\left\{\bar{T}_{\bar{\mu}_{1}}, \bar{T}_{\bar{\mu}_{2}}\right\}$ where $\bar{T}_{\bar{\mu}_{1}}=[1,1]-\operatorname{rsup}\left\{\bar{\mu}_{1}(x): x \in X\right\}$ and $\bar{T}_{\bar{\mu}_{2}}=$ $[1,1]-\operatorname{rsup}\left\{\bar{\mu}_{2}(x): x \in X\right\}$. Then the intersection of $\bar{\alpha}$-translation of $\bar{\mu}_{1}$ and $\overline{\alpha^{\prime}}$-translation of $\bar{\mu}_{2}$ for some $\bar{\alpha}, \overline{\alpha^{\prime}} \in[\overline{0}, \bar{T}]$ is an i-v fuzzy $\beta$-subalgebra of $X$.

Proof. Let $\bar{\mu}_{1}$ and $\bar{\mu}_{2}$ be two i-v fuzzy $\beta$-subalgebra of $X$.

Then by theorem $3.3 \bar{\mu}_{1} \frac{\bar{T}}{\alpha}$ and $\bar{\mu}_{2} \frac{\bar{T}}{\alpha^{\prime}}$ are i-v fuzzy $\beta$-subalgebra of $X$.

For $x, y \in X$,

$$
\begin{aligned}
& \left(\bar{\mu}_{1} \frac{\bar{T}}{\alpha} \cap \bar{\mu}_{2} \frac{\bar{T}}{\alpha^{\prime}}\right)(x+y)=\operatorname{rmin}\left\{\bar{\mu}_{1} \frac{\bar{T}}{\alpha}(x+y), \bar{\mu}_{2} \frac{\bar{T}}{\alpha^{\prime}}(x+y)\right\} \\
& \geq \operatorname{rmin}\left\{\operatorname{rmin}\left\{\bar{\mu}_{1} \frac{\bar{T}}{\alpha}(x), \bar{\mu}_{1 \frac{T}{\alpha}}(y)\right\}, \operatorname{rmin}\left\{\bar{\mu}_{2} \frac{\bar{T}}{\alpha^{\prime}}(x), \bar{\mu}_{2} \frac{\bar{T}}{\alpha^{\prime}}(y)\right\}\right\} \\
& =\operatorname{rmin}\left\{\operatorname{rmin}\left\{\bar{\mu}_{1} \frac{\bar{T}}{\alpha}(x), \bar{\mu}_{2} \frac{\bar{T}}{\alpha^{\prime}}(x)\right\}, \operatorname{rmin}\left\{\bar{\mu}_{1} \frac{\bar{T}}{\alpha}(y), \bar{\mu}_{2} \frac{\bar{T}}{\alpha^{\prime}}(y)\right\}\right\} \\
& =\operatorname{rmin}\left\{\left(\bar{\mu}_{1 \frac{\bar{\alpha}}{\alpha}}^{\bar{T}} \cap \bar{\mu}_{2} \frac{\bar{T}}{\alpha^{\prime}}\right)(x),\left(\bar{\mu}_{1} \frac{\bar{T}}{\alpha} \cap \bar{\mu}_{2} \frac{\bar{T}}{\alpha^{\prime}}\right)(y)\right\} .
\end{aligned}
$$

Similarly

$$
\left(\bar{\mu}_{1} \bar{T}_{\alpha}^{\bar{T}} \cap \bar{\mu}_{2} \frac{\bar{T}}{\alpha^{\prime}}\right)(x-y) \geq \operatorname{rmin}\left\{\left(\bar{\mu}_{1} \frac{\bar{T}}{\alpha} \cap \bar{\mu}_{2} \frac{\bar{T}}{\alpha^{\prime}}\right)(x),\left(\bar{\mu}_{1} \frac{\bar{T}}{\alpha} \cap \bar{\mu}_{2} \frac{\bar{T}}{\alpha^{\prime}}\right)(y)\right\} .
$$

Therefore

$$
\left(\bar{\mu}_{1} \overline{\bar{\alpha}} \cap \bar{\mu}_{2} \frac{\bar{T}}{\alpha^{\prime}}\right)
$$

is an $\mathrm{i}-\mathrm{v}$ fuzzy $\beta$-subalgebra of $X$.

Definition 3.9. Let $f: X \rightarrow Y$ be a function. Let $\bar{\mu}_{X}$ and $\bar{\mu}_{Y}$ be an i-v fuzzy $\bar{\alpha}$-translation on $X$ and $Y$ respectively. Then inverse image of $\bar{\mu}_{Y}$ under $f$ is defined by $f^{-1}\left(\bar{\mu}_{Y}\right)=\left\{f^{-1}\left(\bar{\mu}_{Y}\right) \frac{\bar{T}}{\alpha}(x): x \in X\right\}$ such that $f^{-1}\left(\bar{\mu}_{Y}\right) \frac{\bar{T}}{\alpha}(x)=$ $\bar{\mu}_{Y}(f(x)+\bar{\alpha})$

Theorem 3.10. Let $X$ and $Y$ be two $\beta$-algebras and $f: X \rightarrow Y$ be a homomorphism. If the i-v fuzzy $\bar{\alpha}$-translation $\bar{\mu}_{Y}$ of $Y$ is an $\mathrm{i}-\mathrm{v}$ fuzzy $\beta$-subalgebra of $Y$, then $f^{-1}\left(\bar{\mu}_{Y}\right)$ is an i-v fuzzy $\beta$-subalgebra of $X$. 
Proof. Let the i-v fuzzy $\bar{\alpha}-\operatorname{translation} \bar{\mu}_{Y}$ of $Y$ be an i-v fuzzy $\beta$-subalgebra of $Y$.

Take $x, y \in Y$. Then

$$
\begin{aligned}
f^{-1}\left(\bar{\mu}_{Y} \overline{\bar{\alpha}}\right)(x+y) & =f^{-1}\left(\bar{\mu}_{Y}\right)(x+y)+\bar{\alpha} \\
& =\bar{\mu}_{Y}(f(x+y)+\bar{\alpha}) \\
& =\bar{\mu}_{Y}(f(x)+f(y))+\bar{\alpha} \\
& \geq \operatorname{rmin}\left\{\bar{\mu}_{Y}(f(x)+\bar{\alpha}), \bar{\mu}_{Y}(f(y)+\bar{\alpha})\right\} \\
& =\operatorname{rmin}\left\{f^{-1}\left(\bar{\mu}_{Y} \overline{\frac{T}{\alpha}}\right)(x), f^{-1}\left(\bar{\mu}_{Y} \overline{\bar{\alpha}}\right)(y)\right\}
\end{aligned}
$$

Similarly, $f^{-1}\left(\bar{\mu}_{Y} \frac{\bar{T}}{\alpha}\right)(x-y) \geq \operatorname{rmin}\left\{f^{-1}\left(\bar{\mu}_{Y} \frac{\bar{T}}{\alpha}\right)(x), f^{-1}\left(\bar{\mu}_{Y} \frac{\bar{T}}{\alpha}\right)(y)\right\}$.

Hence $f^{-1}\left(\bar{\mu}_{Y}\right)$ is an i-v fuzzy $\beta$-subalgebra of $X$.

Theorem 3.11. Let $X$ and $Y$ be two $\beta$-algebras and $f: X \rightarrow Y$ be a epimorphism. If the $\mathrm{i}-\mathrm{v}$ fuzzy $\bar{\alpha}$-translation $\bar{\mu}_{X}$ of $X$ is an $\mathrm{i}-\mathrm{v}$ fuzzy $\beta$-subalgebra of $X$, then $f\left(\bar{\mu}_{X}\right)$ is an i-v fuzzy $\beta$-subalgebra of $Y$.

Proof. Let the i-v fuzzy $\bar{\alpha}$-translation $\bar{\mu}_{X}$ of $X$ is an i-v fuzzy $\beta$-subalgebra of $X$.

Take $x, y \in Y$. Then

$$
\begin{aligned}
f\left(\bar{\mu}_{X} \frac{\bar{T}}{\alpha}\right)(x+y) & =f\left(\bar{\mu}_{X}\right)(x+y)+\bar{\alpha} \\
& =\bar{\mu}_{X}(f(x+y)+\bar{\alpha}) \\
& =\bar{\mu}_{X}(f(x)+f(y))+\bar{\alpha} \\
& \geq \operatorname{rmin}\left\{\bar{\mu}_{X}(f(x)+\bar{\alpha}), \bar{\mu}_{X}(f(y)+\bar{\alpha})\right\} \\
& =\operatorname{rmin}\left\{f\left(\bar{\mu}_{X} \overline{\bar{T}}\right)(x), f\left(\bar{\mu}_{X} \overline{\bar{T}}\right)(y)\right\}
\end{aligned}
$$

similarly, $f\left(\bar{\mu}_{X} \frac{\bar{T}}{\alpha}\right)(x-y) \geq \operatorname{rmin}\left\{f\left(\bar{\mu}_{\bar{\alpha}}^{\bar{T}}(x)\right), f\left(\bar{\mu}_{\bar{\alpha}}^{\bar{T}}(y)\right)\right\}$.

Hence $f\left(\bar{\mu}_{X} \frac{\bar{T}}{\alpha}\right)$ is an i-v fuzzy $\beta$-subalgebra of $Y$.

Theorem 3.12. Let $\bar{\mu}_{1}$ and $\bar{\mu}_{2}$ be two i-v fuzzy $\beta$-subalgebras of $X$. Let $\bar{T}=\operatorname{rmin}\left\{\bar{T}_{\bar{\mu}_{1}}, \bar{T}_{\bar{\mu}_{2}}\right\}$ where $\bar{T}_{\bar{\mu}_{1}}=[1,1]-\operatorname{rsup}\left\{\bar{\mu}_{1}(x): x \in X\right\}$ and $\bar{T}_{\bar{\mu}_{2}}=$ $[1,1]-r \sup \left\{\bar{\mu}_{2}(x): x \in X\right\}$. Let $\bar{\alpha} \in[\overline{0}, \bar{T}]$. Then the $\bar{\alpha}-$ translation of cartesian product $\bar{\mu}_{1} \times \bar{\mu}_{2}$ of $\bar{\mu}_{1}$ and $\bar{\mu}_{2}$ is an i-v fuzzy $\beta$-subalgebra of $X \times X$.

Proof. Let $\bar{\mu}_{1}$ and $\bar{\mu}_{2}$ be an i-v fuzzy $\beta$-subalgebra of a $\beta$-algebra $X$ and $\bar{\alpha} \in[\overline{0}, \bar{T}]$.

Now by theorem $3.3 \bar{\mu}_{1} \frac{\bar{T}}{\alpha}$ and $\bar{\mu}_{2} \frac{\bar{T}}{\alpha}$ are i-v fuzzy $\beta$-subalgebra of $X$. 
Clearly $\bar{\mu}_{1} \frac{\bar{T}}{\alpha} \times \bar{\mu}_{2} \frac{\bar{T}}{\alpha}$ is an i-v fuzzy $\beta$-subalgebra of $X \times X$. Also

$$
\begin{aligned}
\left(\bar{\mu}_{1} \times \bar{\mu}_{2}\right) \frac{\bar{T}}{\alpha}(a, b) & =\left(\bar{\mu}_{1} \times \bar{\mu}_{2}\right)(a, b)+\bar{\alpha} \\
& =\operatorname{rmin}\left\{\bar{\mu}_{1}(a), \bar{\mu}_{2}(b)\right\}+\bar{\alpha} \\
& =\operatorname{rmin}\left\{\bar{\mu}_{1}(a)+\bar{\alpha}, \bar{\mu}_{2}(b)+\bar{\alpha}\right\} \\
& =\operatorname{rmin}\left\{\bar{\mu}_{1} \frac{\bar{T}}{\alpha}(a), \bar{\mu}_{2} \bar{\alpha}(b)\right\} \\
& =\left(\bar{\mu}_{1} \overline{\frac{T}{\alpha}} \times \bar{\mu}_{2} \overline{\frac{T}{\alpha}}\right)(a, b)
\end{aligned}
$$

Hence $\left(\bar{\mu}_{1} \times \bar{\mu}_{2}\right) \frac{\bar{T}}{\alpha}$ is an i-v fuzzy $\beta$-subalgebra of $X \times X$

\section{Interval Valued Fuzzy Multiplication of $\beta$-Subalgebra}

In this section, we introduce the notion of interval valued fuzzy $\bar{\phi}$-multiplication. To illustrate the concept, we discuss some examples. Also we prove some simple results.

Definition 4.1. Let $\bar{\mu}$ be an i-v fuzzy subset of $X$ and $\bar{\phi} \in D[0,1]$. A mapping $\bar{\mu}_{\bar{\phi}}^{M}: X \rightarrow D[0,1]$ is said to be an i-v fuzzy $\bar{\phi}$-multiplication of $\bar{\mu}$ if it satisfies $\bar{\mu} \frac{M}{\phi}(x)=\bar{\phi} \cdot \bar{\mu}(x) \quad \forall x \in X$

Example 4.2. Consider the above example 3.2. Let $\bar{\phi}=[0.2,0.3]$. Then the $\bar{\phi}$-multiplication of $\mathrm{i}-\mathrm{v}$ fuzzy set $\bar{\mu}$ is given by

$\bar{\mu}_{\bar{\phi}}^{M}(0)=[0.06,0.21], \quad \bar{\mu}_{\bar{\phi}}^{M}(a)=\bar{\mu}_{\bar{\phi}}^{M}(c)=[0.02,0.15]$ and $\bar{\mu}_{\bar{\phi}}^{M}(b)=[0.04,0.18]$.

Theorem 4.3. For any i-v fuzzy $\beta$-subalgebra $\bar{\mu}$ of $X$ and $\bar{\phi} \in D[0,1]$, the $\mathrm{i}-\mathrm{v}$ fuzzy $\bar{\phi}$-multiplication $\bar{\mu}_{\bar{\phi}}^{M}(x)$ of $\bar{\mu}$ is an i-v fuzzy $\beta$-subalgebra of $X$.

Proof. Let $x, y \in X$ and $\bar{\phi} \in D[0,1]$, Then

$$
\bar{\mu}(x+y) \geq \operatorname{rmin}\{\bar{\mu}(x), \bar{\mu}(y)\}
$$

and

$$
\bar{\mu}(x-y) \geq \operatorname{rmin}\{\bar{\mu}(x), \bar{\mu}(y)\}
$$

Now

$$
\begin{aligned}
\bar{\mu}_{\bar{\phi}}^{M}(x+y) & =\bar{\phi} \cdot \bar{\mu}(x+y) \\
& \geq \bar{\phi} \cdot \operatorname{rmin}\{\bar{\mu}(x), \bar{\mu}(y)\}
\end{aligned}
$$




$$
\begin{aligned}
& =\operatorname{rmin}\{\bar{\phi} \cdot \bar{\mu}(x), \bar{\phi} \cdot \bar{\mu}(y)\} \\
& =\operatorname{rmin}\left\{\bar{\mu} \frac{M}{\phi}(x), \bar{\mu} \frac{M}{\phi}(y)\right\}
\end{aligned}
$$

Similarly, $\bar{\mu}_{\bar{\phi}}^{M}(x-y) \geq \operatorname{rmin}\left\{\bar{\mu}_{\bar{\phi}}^{M}(x), \bar{\mu}_{\bar{\phi}}^{M}(y)\right\}$.

Hence $\bar{\mu} \frac{M}{\phi}$ of $\bar{\mu}$ is a i-v fuzzy $\beta$-subalgebra of $X$.

The following is the converse of the above theorem.

Theorem 4.4. For any i-v fuzzy subset $\bar{\mu}$ of $X$ and $\bar{\phi} \in D[0,1]$. If the i-v fuzzy $\bar{\phi}$-multiplication $\bar{\mu} \frac{M}{\phi}$ of $\bar{\mu}$ is also an i-v fuzzy $\beta$-subalgebra of $X$, then so is $\bar{\mu}$.

Proof. Let $x, y \in X$. Assume that $\bar{\mu}_{\bar{\phi}}^{M}(x)$ of $\bar{\mu}$ is a i-v fuzzy $\beta$-subalgebra of $X$ for some $\bar{\phi} \in D[0,1]$.

Then

$$
\begin{aligned}
\bar{\phi} \cdot \bar{\mu}(x+y) & =\bar{\mu}_{\bar{\phi}}^{M}(x+y) \\
& \geq \operatorname{rmin}\left\{\bar{\mu}_{\bar{\phi}}^{M}(x), \bar{\mu}_{\bar{\phi}}^{M}(y)\right\} \\
& =\operatorname{rmin}\{\bar{\phi} \cdot \bar{\mu}(x), \bar{\phi} \cdot \bar{\mu}(y)\} \\
& =\bar{\phi} \cdot \operatorname{rmin}\{\bar{\mu}(x), \bar{\mu}(y)\}
\end{aligned}
$$

$\Rightarrow \bar{\mu}(x+y) \geq \operatorname{rmin}\{\bar{\mu}(x), \bar{\mu}(y)\}$.

Similarly, $\bar{\mu}(x-y) \geq \operatorname{rmin}\{\bar{\mu}(x), \bar{\mu}(y)\}$.

Hence $\bar{\mu}$ is an i-v fuzzy $\beta$-subalgebra of $X$.

Definition 4.5. Let $\bar{\mu}$ be an i-v fuzzy subset of $X, \bar{\phi} \in D[0,1]$ and $\bar{\alpha} \in[\overline{0}, \bar{T}]$. A mapping $\bar{\mu}_{\bar{\phi} \bar{\alpha}}^{M \bar{T}}: X \rightarrow D[0,1]$ is said to be an i-v fuzzy magnified $-\bar{\phi} \bar{\alpha}$-translation of $\bar{\mu}$ if it satisfies $\bar{\mu}_{\bar{\phi} \bar{\alpha}}^{M \bar{T}}(x)=\bar{\phi} \cdot \bar{\mu}(x)+\bar{\alpha} \quad \forall x \in X$.

Example 4.6. Consider the $\beta$-algebra $X=\{0, a, b, c\}$ in example 2.2. Define an interval valued fuzzy subset $\bar{\mu}$ of $X$ by

$$
\bar{\mu}(x)= \begin{cases}{[0.3,0.7]:} & x=0 \\ {[0.1,0.5]:} & x=a, c \\ {[0.2,0.6]:} & x=b\end{cases}
$$

Then $\bar{\mu}$ is an i-v fuzzy $\beta$-subalgebra of $X$. Here $\bar{T}=[1,1]-\operatorname{rsup}\{\bar{\mu}(x) / x \in$ $X\}=[1,1]-[0.3,0.7]=[0.7,0.3]$. choose $\bar{\alpha}=[0.04,0.08] \in[[0,0],[0.7,0.3]$ and $\bar{\phi} .=[0.1,0.3] \in D[0,1]$. 
Then the i-v fuzzy set $\bar{\mu}_{\bar{\phi} \bar{\alpha}}^{M \bar{T}}: X \rightarrow D[0,1]$ is given by

$$
\bar{\mu} \frac{M \bar{T}}{\bar{\phi}}(0)=[0.07,0 . .29], \quad \bar{\mu}_{\bar{\phi}}^{M \bar{\alpha}}(a)=\bar{\mu}_{\bar{\phi}}^{M \bar{\alpha}}(c)=[0.05,0.23]
$$

and

$$
\bar{\mu}_{\bar{\phi} \bar{\alpha}}^{M \bar{T}}(b)=[0.06,0.26] .
$$

Theorem 4.7. Let $\bar{\mu}$ be an i-v fuzzy subset of $X, \bar{\phi} \in D[0,1]$ and $\bar{\alpha} \in[\overline{0}, \bar{T}]$. A mapping $\bar{\mu}_{\bar{\phi} \bar{\alpha}}^{M \bar{T}}: X \rightarrow D[0,1]$ is an i-v fuzzy magnified- $\bar{\phi} \bar{\alpha}$-translation of $\bar{\mu}$. Then $\bar{\mu}$ is an i-v fuzzy $\beta$-subalgebra of $X$ if and only if $\bar{\mu}_{\bar{\phi}}^{M \bar{\alpha}}$ is an i-v fuzzy $\beta$-subalgebra of $X$.

Proof. Let $\bar{\mu}$ be an i-v fuzzy subset of $X, \bar{\phi} \in D[0,1]$ and $\bar{\alpha} \in[\overline{0}, \bar{T}]$. A mapping $\bar{\mu}_{\bar{\phi} \bar{\alpha}}^{M \bar{T}}: X \rightarrow D[0,1]$ is said to be an i-v fuzzy magnified- $\bar{\phi} \bar{\alpha}$-translation of $\bar{\mu}$.

Assume that $\bar{\mu}$ is an $\mathrm{i}-\mathrm{v}$ fuzzy $\beta$-subalgebra of $X$.

Then $\bar{\mu}(x+y) \geq \operatorname{rmin}\{\bar{\mu}(x), \bar{\mu}(y)\}$ and $\bar{\mu}(x-y) \geq \operatorname{rmin}\{\bar{\mu}(x), \bar{\mu}(y)\}$.

Now

$$
\begin{aligned}
\bar{\mu}_{\bar{\phi} \bar{\alpha}}^{M \bar{T}}(x+y) & =\bar{\phi} \cdot \bar{\mu}(x+y)+\bar{\alpha} \\
& \geq \bar{\phi} \cdot \operatorname{rmin}\{\bar{\mu}(x), \bar{\mu}(y)\}+\bar{\alpha} \\
& =\operatorname{rmin}\{\bar{\phi} \cdot \bar{\mu}(x)+\bar{\alpha}, \bar{\phi} \cdot \bar{\mu}(y)+\bar{\alpha}\} \\
& =\operatorname{rmin}\left\{\bar{\mu}_{\bar{\phi} \bar{\alpha}}^{M \bar{T}}(x), \bar{\mu}_{\bar{\phi}}^{M \bar{\alpha}}(y)\right\}
\end{aligned}
$$

Similarly, $\bar{\mu}_{\bar{\phi} \bar{\alpha}}^{M \bar{T}}(x-y) \geq \operatorname{rmin}\left\{\bar{\mu}_{\bar{\phi} \bar{\alpha}}^{M \bar{T}}(x), \bar{\mu}_{\bar{\phi} \bar{\alpha}}^{M \bar{T}}(y)\right\}$.

Hence $\bar{\mu} \frac{M \bar{T}}{\bar{\alpha}}$ of $\bar{\mu}$ is an i-v fuzzy $\beta$-subalgebra of $X$.

Assume that $\bar{\mu}_{\bar{\phi}}^{M \bar{\alpha}}(x)$ of $\bar{\mu}$ is an i-v fuzzy $\beta$-subalgebra of $X$

Then:

$$
\begin{aligned}
\bar{\phi} \cdot \bar{\mu}(x+y)+\bar{\alpha} & =\bar{\mu}_{\bar{\phi} \bar{\alpha}}^{M \bar{T}}(x+y) \\
& \geq \operatorname{rmin}\left\{\bar{\mu} \frac{M \bar{\phi} \bar{\alpha}}{\bar{\alpha}}(x), \bar{\mu} \bar{\phi} \bar{\alpha}\right. \\
& =\operatorname{rmin}\{\bar{\phi} \cdot \bar{\mu}(x)+\bar{\alpha}, \bar{\phi} \cdot \bar{\mu}(y)+\bar{\alpha}\} \\
& =\bar{\phi} \cdot \operatorname{rmin}\{\bar{\mu}(x), \bar{\mu}(y)\}+\bar{\alpha}
\end{aligned}
$$

$\Rightarrow \bar{\mu}(x+y) \geq \operatorname{rmin}\{\bar{\mu}(x), \bar{\mu}(y)\}$.

Similarly, $\bar{\mu}(x-y) \geq \operatorname{rmin}\{\bar{\mu}(x), \bar{\mu}(y)\}$.

Hence $\bar{\mu}$ is an i-v fuzzy $\beta$-subalgebra of $X$. 


\section{References}

[1] M. Aub Ayub Anasri, M. Chandramouleeswaran, Fuzzy translations of fuzzy $\beta$-subalgebra of $\beta$-algebras, International Journal of Pure and Applied Mathematics, 92, No. 5 (2014), 657-667.

[2] S.R. Barbhuiya, Fuzzy translation and fuzzy multiplications of interval valued fuzzy BGalgebras, International Journal of Mathematical Archive, 7, No. 6 (2015), 25-32.

[3] M. Chandramouleeswaran, P. Muralikrishna, S. Srinivasan, Fuzzy translation and fuzzy multiplication in BF/BG-algebras, Indian Journal of Science and Technology, 6, No. 9 (2013), 5216-5219, doi: 10.17485/ijst/2013/v6i9/37135.

[4] P. Hemavathi, P. Muralikrishna, K. Palanivel, A note on interval valued fuzzy $\beta$ subalgebras, Global Journal of Pure and Applied Mathematics, 11, No. 4 (2015), 25532560 .

[5] P. Hemavathi, P. Muralikrishna, K. Palanivel, Product on i-v fuzzy $\beta$-subalgebra, Accepted for publication.

[6] K. Iseki, S. Tanaka, An introduction to theory of BCK-algebras, Math. Japon., 23 (1973), $1-26$.

[7] J. Neggers, Kim Hee Sik, On $\beta$-algebras, Math. Slovaca., 52, No. 5 (2002), 517-530.

[8] K. Sujatha, P. Muralikrishna, Intuitionistic fuzzy $\alpha$-Translation on $\beta$-algebras, International Journal of Pure and Applied Mathematics, 98, No. 5 (2015), 39-44, doi: 10.12732/ijpam.v98i5.7.

[9] L.A. Zadeh, Fuzzy sets, Inform. Control, 8, No. 3 (1965), 338-353, doi: 10.1016/S00199958(65)90241-X.

[10] L.A. Zadeh, The concept of a linguistic variable and its application to approximate reasoning, I, Information Sci., 8 (1975), 199-249, doi: 10.1016/0020-0255(75)90036-5. 